\title{
LEVANTAMENTO FLORÍSTICO DAS MUDAS DE RESTINGA PRODUZIDAS NO HORTO RESTINGA NO MUNICÍPIO DO RIO DE JANEIRO, RJ, BRASIL
}

\section{FLORISTIC INVENTORY OF RESTINGAS SEEDLINGS PRODUCED IN THE HORTO RESTINGA IN RIO DE JANEIRO CITY, RJ, BRAZIL}

\author{
Janice Rezende Vieira Peixoto \\ Universidade Federal Fluminense - UFF \\ Carlos José Ruffato Favoreto \\ Environ Consultoria e Projetos Limitada
}

Allana Oliveira Lima

Universidade Federal Fluminense - UFF

Bianca Alves Lima Ribeiro

Universidade do Estado do Rio de Janeiro - UERJ

\section{RESUMO}

As restingas do município do Rio de Janeiro, RJ, estão cada vez mais sofrendo com os impactos ambientais oriundos de atividades antrópicas, logo, é de suma importância a produção de mudas de restinga para a recuperação de áreas degradadas e para reflorestamento visando 0 cumprimento de medidas compensatórias. No Horto restinga foram registradas 47.390 mudas, as quais todas são nativas e cerca de $55 \%$ das espécies observadas são consideradas endêmicas do Brasil. Dentre as 75 espécies identificadas, 15 estão categorizadas segundo o grau de ameaça de extinção nas fontes consultadas. Destaca-se a espécie Inga maritima Benth, endêmica das restingas do Rio de Janeiro, que é classificada como ameaçada de extinção em todas as listas analisadas, devido à degradação do seu habitat que já é naturalmente restrito.

Palavras-Chaves: Mudas nativas, Inga marítima, espécies de restinga.

\section{ABSTRACT}

The Restingas of Rio de Janeiro Municipality, RJ, are increasingly suffering with environmental impacts arising from human activities. Therefore, it is extremely important to produce Restingas seedlings to land reclamation and reforestation aimed at meeting of compensatory measures. In the Horto Restinga, 47,390 seedlings were recorded, which are all native and $54.67 \%$ of the species observed are endemic in

Revista Internacional de Ciências · v.5 - n.2 · jul./dez. 2015 
Brazil. Among the 75 species identified, 15 are categorized according to the degree of threat of extinction in the sources consulted. It is essential highlight Inga maritima Benth, a specie endemic to the Restingas of Rio de Janeiro, that is classified as endangered in all lists analyzed due to degradation of their habitat which is already naturally restricted.

Key words: Native seedlings, Inga maritima, species of Restinga

\section{INTRODUÇÃO}

Nos últimos anos, as restingas do município do Rio de Janeiro estão sofrendo impactos ambientais decorrentes de ações antrópicas, influenciados pela pressão imobiliária de diversos bairros do município. É de grande importância a conservação dos remanescentes existentes e a restauração de áreas degradadas, portanto,a produção de mudas é etapa fundamental neste processo (ZAMITH; SCARANO, 2004).

Segundo a Resolução CONAMA 303 (2002), a restinga é um depósito arenoso paralelo a linha da costa que apresenta forma alongada, produzida por processos de sedimentação, são consideradas comunidades edáficas por dependerem mais da natureza do solo que do clima.

As restingas podem ser descritas como um grupo de ecossistemas que abrange comunidades florísticas e fisionomicamente distintas, localizados em terrenos de idade quaternária, podendo apresentar origem fluvial, lagunar, eólica ou combinações destas, com solos, no geral, pouco desenvolvidos. Formam um característico complexo vegetacional encontrado em praias, cordões arenosos, dunas e depressões associadas (FALKENBERG, 1999).

A restinga é caracterizada por uma vegetação bastante heterogênea, possui desde formações herbáceas, passando por formações arbustivas, chegando a florestas cuja altura máxima do dossel não é superior a $20 \mathrm{~m}$ de altura (SILVA, 1999; GOMES et al., 2007). Suas comunidades são distribuídas em mosaico, apresentam poucos tipos próprios de vegetação, aliás, é muito comum, as espécies encontradas neste ambiente serem oriundas de outras comunidades (RIZZINI, 1997; OLIVEIRA, 2014), essas espécies habitam as restingas por causa da diversidade das condições físicas locais, podendo 
ocasionar variações fisionômicas em razão das condições distintas de seu ambiente de origem (ASSUMPÇÃO; NASCIMENTO, 2000).

De acordo com Carrasco et al. (2012), é provável que as florestas de restingas sejam um dos ecossistemas mais ameaçados em função da pressão antrópica, que causa o corte raso de grandes áreas de vegetação e alterações no substrato, para posterior ocupação imobiliária, invasão de espécies exóticas, extrativismo seletivo de espécies florestais e atividades de mineração. Além disso, este ecossistema ocupa uma pequena extensão da faixa territorial o que o torna ainda mais vulnerável ao desaparecimento.

Nos últimos anos tem ocorrido grande demanda por mudas de espécies nativas, considerando que estas espécies são fundamentais em plantios visando restauração florestal (BARBOSA et al., 2003). Entre os anos de 2009 e 2012, aproximadamente 175 projetos de implantação de recuperação de áreas degradadas foram analisados pelo órgão ambiental estadual, perfazendo aproximadamente 14 mil hectares de áreas a serem recuperadas em todo o estado do Rio de Janeiro (SILVEIRA-FILHO, 2012; ALONSO et al., 2014). Vale salientar que em função das diversas construções olímpicas e obras de mobilidade realizadas na cidade do Rio de Janeiro, assim como a implantação de diversos empreendimentos ao longo dos próximos anos reforça a necessidade de produção de mudas nativas para serem utilizadas em reflorestamentos e cumprimento de medidas compensatórias. Apesar da grande importância da restauração de restingas do município, há poucos hortos com produção exclusiva de mudas de espécies características de restinga.

Um viveiro de produção de mudas deve produzir diferentes espécies florestais dos diversos grupos sucessionais, contemplando aspectos relacionados ao uso de espécies nativas de ocorrência regional e reproduzindo a diversidade genética e florística da região (RODRIGUES, 2000). O sucesso de um reflorestamento é diretamente condicionado pela qualidade das mudas produzidas, estas devemresistir às condições adversas encontradas no campo (GOMES et al.1991).

Este trabalho tem como principal objetivo realizar um levantamento florístico das mudas produzidas no Horto Restinga, buscando analisar a diversidade de espécies do horto. 


\section{MATERIAL E MÉTODOS}

\section{1. Área de Estudo}

O Horto Restinga está localizado no bairro da Barra da Tijuca, município do Rio de Janeiro em que o acesso é feito pela Rua General Moisés Castelo Branco Filho. O horto encontra-se entre as coordenadas de latitude $23^{\circ} 0^{\prime} 12.29^{\prime \prime}$ a $23^{\circ} 0^{\prime} 18.23^{\prime \prime S}$ e longitude $43^{\circ} 24^{\prime} 2.57^{\prime \prime}$ a 4324'0.16"O, foi criado no ano 2010.

Segundo os dados do Instituto Nacional de Meteorologia (INMET), o município do Rio de Janeiro apresenta temperatura média de $23,7^{\circ} \mathrm{C}$, precipitação média anual de $1.171 \mathrm{~mm}$ e média anual de umidade relativa do ar de $79 \%$, para o período entre 1973 e 1990.

Como sugere o próprio nome, o horto tem como principal objetivo a produção de mudas das espécies características de restinga para serem plantadas no Campo de Golfe Olímpico, conforme descrito no Plano de Recuperação de Áreas Degradas (PRAD) elaborado com a finalidade de minimizar os possíveis impactos ambientais gerados ao longo da implantação e durante o funcionamento do empreendimento em questão. Portanto, o plantio das mudas busca uma recomposição ecológica e estrutural das restingas do município do Rio de Janeiro.

Realiza-se no horto tanto a fase de: produção de mudas em viveiro de semeadura, como a fase de condução e rustificação em embalagens. A área de produção de mudas tem aproximadamente $4.000 \mathrm{~m}^{2} \mathrm{e}$ a área construída é de $60 \mathrm{~m}^{2}$.

\section{Censo e Análise Florística}

Realizou-se o censo das mudas do Horto Restinga, durante o mês de agosto de 2015, no qual foi considerada todas as mudas que apresentavam qualidade satisfatória para serem encaminhadas para o Campo de Golfe Olímpico, foram contabilizadas mudas de diversos estágios de crescimento e desenvolvimento. É importante destacar que foram contabilizadas somente as 
mudas presentes no horto durante o período do censo, não considerando assim, as mudas que já haviam sido plantadas no Campo de Golfe Olímpico.

Para a classificação de famílias botânicas, nomes científicos, origem, endemismo e forma de vida das espécies registradas, adotou-se a Lista de Espécies da Flora do Brasil elaborado pelo Jardim Botânico do Rio de Janeiro.

A verificação do nível de ameaça de extinção das espécies encontradas, foi realizada através de consulta das seguintes listas: Lista Oficial de Espécies Ameaçadas de Extinção do Município do Rio de Janeiro (Secretaria Municipal de Meio Ambiente do Rio de Janeiro - SMAC, 2000); Lista Nacional Oficial de Espécies da Flora Ameaçadas de Extinção (Ministério do Meio Ambiente MMA, 2014);Livro Vermelho da Flora do Brasil (Instituto de Pesquisas Jardim Botânico do Rio de Janeiro, 2013); e Lista Vermelha de Espécies Ameaçadas de Extinção da União Internacional para a Conservação da Natureza - IUCN (International Union for Conservation of Nature, 2015).

As categorias de ameaça adotadas nas listas são ordenadas com base no grau descrescente de ameaça: Extinta (EX), Extinta na Natureza (EW), Criticamente Ameaçada (CR), Ameaçada (EN), Vulnerável (VU), Baixo Risco (LR).

\section{RESULTADOS E DISCUSSÃO}

\section{Caracterização da Flora do Horto Restinga}

No Horto Restinga foram contabilizadas 47.390 mudas aptas para serem utilizadas em reflorestamentos. Identificou-se 78 espécies, representadas por 64 gêneros, distribuídas em 30 famílias botânicas. No entanto, 05 espécies só foram identificadas em nível de gênero. As cinco famílias que apresentaram maiores riqueza de espécies foram Fabaceae (13), Myrtaceae (11), Arecaceae (5), Bromeliaceae (4) e Clusiaceae (4), conforme listado na Tabela 1. Juntas, essas famílias, representam $47,43 \%$ do total de espécies registradas no horto. As famílias Fabaceae e Myrtaceae costumam ser as mais representativas nas restingas do estado do Rio de Janeiro, como observado por: Assumpção e Nascimento (2000) no Complexo Lagunar Grussaí/lquipari em São João da 
Barra- RJ; Pereira et al (2001) na restinga de Barra de Maricá - RJ, Afonso et al. (2007) na Restinga da Marambaia - RJ, Ribeiro e Lima (2009) para o Centro de Diversidade Vegetal de Cabo Frio

É importante destacar que todas as espécies produzidas no horto são nativas e cerca de $55 \%$ das espécies observadas são consideradas endêmicas do Brasil. A grande variedade de espécies endêmicas registradas no horto, evidenciam a importância da produção destas mudas visando o reflorestamento de áreas afetadas por atividades antrópicas. Conforme Kruckeberg e Rabnowitz (1985) e Gentry (1986) afirmaram, o grau de endemismo e raridade de uma localidade são importantes critérios para determinar áreas com potencial para conservação e devem ser uma preocupação nos estudos de floras regionais.

Das plantas registradas no horto, 57 espécies apresentam como principal forma de dispersão das sementes a zoocoria, 09 são anemocóricas, 05 autocóricas e as demais apresentam mais de um tipo de síndrome de dispersão ou não foram definidas. Em restingas e florestas tropicais é comum a predominância de espécies zoocóricas (ALVES; METZGER, 2006; RODRIGUES et al, 2010).

Tabela 1: Listagem das famílias, espécies e nomes populares, síndromes de dispersão, forma de vida e quantidade das plantas registradas no Horto Restinga. Síndrome de dispersão: Zoo Zoocórica; Aut - Autocórica; Ane - Anemocórica. Forma de vida: Arb/Arv - Arbusto/ Árvore; Lia/ Vol/ Trep - Liana/Volúvel/Trepadeira.

\begin{tabular}{|c|c|c|c|c|}
\hline Família/ Espécie & Nome popular & $\begin{array}{c}\text { Síndrome de } \\
\text { dispersão }\end{array}$ & $\begin{array}{l}\text { Forma de } \\
\text { Vida }\end{array}$ & Quantidad \\
\hline \multicolumn{5}{|l|}{ ANACARDIACEAE } \\
\hline Schinus terebinthifolius Raddi & Aroeira & Zoo & Arb/Arv & 126 \\
\hline $\begin{array}{c}\text { Tapirira guianensis Aubl. } \\
\text { ANNONACEAE }\end{array}$ & Feijão de campo & Zoo & Árvore & 1146 \\
\hline Annona glabra L. & Pinha-do-brejo & Zoo & Arb/Arv & 347 \\
\hline \multicolumn{5}{|l|}{ APOCYNACEAE } \\
\hline $\begin{array}{c}\text { Aspidosperma parvifolium } \\
\text { A.D.C. }\end{array}$ & Guatambu & Ane & Árvore & 309 \\
\hline \multicolumn{5}{|l|}{ ARECACEAE } \\
\hline $\begin{array}{c}\text { Allagoptera arenaria (Gomes) } \\
\text { Kuntze }\end{array}$ & Guriri & Zoo & Erva & 354 \\
\hline Bactris setosa Mart. & $\begin{array}{l}\text { Palmeira-coco-de- } \\
\text { natal }\end{array}$ & Zoo & Erva & 60 \\
\hline
\end{tabular}


Desmoncus sp.

Euterpe oleracea Mart.

Syagrus romanzoffiana

(Cham.) Glassman

Açal

Zoo

Erva

7

BIGNONIACEAE

Fridericia conjugata (Vell.) L.G.Lohmann

Jerivá

Zoo

Erva

443

Handroanthus chrysotrichus

Arrabide rosa

Ane

Lia/vol/ter

696

Ipê dourado

Ane

Árvore

808

BORAGINACEAE

Cordia superba Cham.

Babosa branca

Zoo

Arb/Arv

1546

Varronia curassavica Jacq.

Erva baleeira

Zoo

Arbusto

14

BROMELIACEAE

Alcantarea sp.

Bromélia

928

Billbergia amoena (Lodd.) Lindl.

Guaricana-uva

Zoo

Erva

8

Bromelia antiacantha Bertol.

Gravatá

Zoo

Erva

712

Vriesea neoglutinosa Mez

Bromélia

Ane

Erva

5

BURSERACEAE

Protium icicariba (DC.) Marchand

Incenso

Zoo

Árvore

613

CAPPARACEAE

Capparis flexuosa (L.) L.

Feijão-bravo

Zoo

Arb/Arv

392

CELASTRACEAE

Couepia ovalifolia (Schott)

Benth. ex Hook.f.

Oiti-da-praia

Arb/Arv

112

Maytenus obtusifolia Mart.

Carne-de-anta

Arb/Arv

3106

CLUSIACEAE

Clusia fluminensis Planch. \& Triana

Clusia

Zoo

Árvore

2505

Clusia lanceolata Cambess.

Garcinia gardneriana (Planch.

\& Triana) Zappi

Abaneiro

Zoo

Arb/Arv

671

Bacupari

Zoo

Arb/Arv

36

Kielmeyera membranacea

Casar.

Ane

Árvore

127

CONVOLVULACEAE

Ipomea pes-caprae (L.) Sweet

Salsa-da-praia

Ane

Erva

484

ERYTHROXYLACEAE

Erythroxylum ovalifolium Peyr. Fruta-de-pomba

Zoo

Arbusto

733

EUPHORBIACEAE

Alchornea

Alchornea
triplinervia (Spreng.) Müll.Arg.

Pau-óleo

Zoo

Arb/Arv

104

Joanesia princeps Vellozo

Cutieira

Zoo

Árvore

37

Sapium glandulosum (L.)

Morong

Leiteiro

Zoo

Arb/Arv

4

FABACEAE 
Abarema sp.

\begin{tabular}{|c|c|c|c|c|}
\hline Andira legalis (Vell.) Toledo & Angelim coco & Zoo & Árvore & 20 \\
\hline Inga laurina (Sw.) Willd. & Ingá da praia & Zoo & Árvore & 1100 \\
\hline Inga maritima Benth. & Ingá & Zoo & Arb/Arv & 936 \\
\hline $\begin{array}{c}\text { Inga subnuda Salzm. ex } \\
\text { Benth. }\end{array}$ & Ingá & Zoo & Árvore & 1090 \\
\hline Inga sp. & & & & 80 \\
\hline $\begin{array}{l}\text { Machaerium lanceolatum } \\
\text { (Vell.) J.F.Macbr. }\end{array}$ & & Ane & Árvore & 722 \\
\hline Ormosia arborea (Vell.) Harms & Olho-de-cabra & Aut/ Zoo & Árvore & 1296 \\
\hline Pithecolobium tortum Mart. & Jurema & Aut & Árvore & 1065 \\
\hline $\begin{array}{c}\text { Senna appendiculata (Vogel) } \\
\text { Wiersema } \\
\text { Senna pendula (Humb. \& }\end{array}$ & $\begin{array}{l}\text { Fedegoso de } \\
\text { restinga }\end{array}$ & Zoo/Ane & Arbusto & 61 \\
\hline $\begin{array}{c}\text { Bonpl.ex Willd.) H.S.Irwin \& } \\
\text { Barneby }\end{array}$ & Canudo-de-pito & Aut & Arb/Arv & 689 \\
\hline Sophora tomentosa L. & Feijão-da-praia & Aut & Arbusto & 227 \\
\hline $\begin{array}{c}\text { Zollernia glabra (Spreng.) } \\
\text { Yakovlev }\end{array}$ & Pau-santo & Zoo & Árvore & 45 \\
\hline \multicolumn{5}{|l|}{ HUMIRIACEAE } \\
\hline $\begin{array}{c}\text { Humiria balsamifera (Aubl.) } \\
\text { J.St.-Hil. }\end{array}$ & & Zoo & Arb/Arv & 2 \\
\hline \multicolumn{5}{|l|}{ LAURACEAE } \\
\hline Cinnamomum sp. & & & & 6 \\
\hline \multicolumn{5}{|l|}{ MALPIGHIACEAE } \\
\hline Byrsonima sericea DC. & Murici & Zoo & Árvore & 663 \\
\hline $\begin{array}{c}\text { Heteropterys coleoptera A. } \\
\text { Juss. }\end{array}$ & & Zoo & Lia/vol/ter & 581 \\
\hline \multicolumn{5}{|l|}{ MALVACEAE } \\
\hline $\begin{array}{c}\text { Ceiba speciosa (A.St.-Hil.) } \\
\text { Ravenna }\end{array}$ & Paineira-rosa & Ane & Árvore & 1164 \\
\hline $\begin{array}{l}\text { Pseudobombax grandiflorum } \\
\text { (Cav.) A.Robyns }\end{array}$ & Imbiruçu & Ane & Árvore & 455 \\
\hline \multicolumn{5}{|l|}{ MELASTOMATACEAE } \\
\hline $\begin{array}{c}\text { Miconia cinnamomifolia (DC.) } \\
\text { Naudin }\end{array}$ & Jacatirão & Zoo & Arb/Arv & 3 \\
\hline \multicolumn{5}{|l|}{ MORACEAE } \\
\hline $\begin{array}{c}\text { Ficus adhatodifolia Schott in } \\
\text { Spreng. }\end{array}$ & Figueira-branca & Zoo & Árvore & 312 \\
\hline Ficus clusiifolia Schott & Figueira-vermelha & Zoo & Árvore & 2 \\
\hline Ficus hirsuta Schott & Figueira & Zoo & Árvore & 33 \\
\hline \multicolumn{5}{|l|}{ MYRTACEAE } \\
\hline $\begin{array}{c}\text { Calyptranthes brasiliensis } \\
\text { Spreng. }\end{array}$ & Guamirim-branco & Zoo & Árvore & 2450 \\
\hline Eugenia brasiliensis Lam. & Grumixama & Zoo & Árvore & 954 \\
\hline Eugenia selloi B.D.Jacks. & Pitangão & Zoo & Arb/Arv & 366 \\
\hline Eugenia punicifolia (Kunth) & Murtinha & Zoo & Arbusto & 1613 \\
\hline
\end{tabular}


DC.

\begin{tabular}{|c|c|c|c|c|}
\hline \multirow{2}{*}{$\begin{array}{c}\text { Eugenia astringens Cambess. } \\
\text { Eugenia sulcata Spring ex } \\
\text { Mart. }\end{array}$} & Aperta-goela & Zoo & Árvore & 768 \\
\hline & Pitanga-preta & Zoo & Árvore & 1574 \\
\hline Eugenia uniflora L. & Pitanga & Zoo & Arbusto & 1128 \\
\hline $\begin{array}{c}\text { Myrcia racemosa (O.Berg) } \\
\text { Kiaersk. }\end{array}$ & Batitô & Zoo & Árvore & 1022 \\
\hline $\begin{array}{c}\text { Myrrhinium atropurpureum } \\
\text { Schott }\end{array}$ & Mirtilo & Zoo & Arb/Arv & 7 \\
\hline $\begin{array}{c}\text { Neomitranthes obscura (DC.) } \\
\text { N.Silveira }\end{array}$ & $\begin{array}{l}\text { Araçá- } \\
\text { preto/Cambuí }\end{array}$ & Zoo & Árvore & 2156 \\
\hline Psidium cattleianum Sabine & Araçá & Zoo & Árvore & 791 \\
\hline \multicolumn{5}{|l|}{ OCHNACEAE } \\
\hline $\begin{array}{c}\text { Ouratea cuspidata (A.St.-Hil.) } \\
\text { Engl. }\end{array}$ & Jabotapita & Zoo & Árvore & 492 \\
\hline \multicolumn{5}{|l|}{ PRIMULACEAE } \\
\hline $\begin{array}{l}\text { Myrsine coriacea(Sw.) R.Br. } \\
\text { ex Roem. \& Schult. }\end{array}$ & Capororoca & Zoo & Arb/Arv & 50 \\
\hline Myrsine umbellata Mart. & Capororoca & Zoo & Árvore & 915 \\
\hline \multicolumn{5}{|l|}{ RUBIACEAE } \\
\hline $\begin{array}{c}\text { Psychotria brachyceras } \\
\text { Müll.Arg. }\end{array}$ & Cafezinho & Zoo & Arbusto & 1665 \\
\hline \multicolumn{5}{|l|}{ RUTACEAE } \\
\hline \multicolumn{5}{|l|}{ SAPINDACEAE } \\
\hline $\begin{array}{l}\text { Allophylus puberulus } \\
\text { (Cambess.) Radlk. }\end{array}$ & Fruta-de-saíra & Zoo & Arbusto & 323 \\
\hline $\begin{array}{l}\text { Cupania emarginata } \\
\text { Cambess. }\end{array}$ & $\begin{array}{l}\text { Camboatá-de- } \\
\text { restinga }\end{array}$ & Zoo & Arb/Arv & 1002 \\
\hline Paullinia coriacea Casar. & Fruta-de-café & Zoo & Arbusto & 88 \\
\hline \multicolumn{5}{|l|}{ SAPOTACEAE } \\
\hline $\begin{array}{c}\text { Manilkara subsericea (Mart.) } \\
\text { Dubard }\end{array}$ & $\begin{array}{l}\text { Massaranduba-da- } \\
\text { praia }\end{array}$ & Zoo & Arb/Arv & 1530 \\
\hline $\begin{array}{c}\text { Pouteria grandiflora (A.DC.) } \\
\text { Baehni }\end{array}$ & Guapéva & Aut & Árvore & 89 \\
\hline $\begin{array}{c}\text { Pouteria venosa (Mart.) } \\
\text { Baehni }\end{array}$ & Abiuzinho & Zoo & Arb/Arv & 22 \\
\hline URTICACEAE & & & & \\
\hline $\begin{array}{c}\text { Cecropia pachystachya Trécul } \\
\text { VERBENACEAE }\end{array}$ & Embaúba & Zoo & Árvore & 4 \\
\hline Vitex polygama Cham. & Maria-preta & Zoo & Arb/Arv & 274 \\
\hline
\end{tabular}

As cinco espécies mais abundantes no horto, ou seja, que apresentaram um número maior de mudas são: Maytenus obtusifolia (3.106), Clusia fluminensis (2505), Calyptranthes brasiliensis (2.450), Neomitranthes obscura (2.156) e Psychotria brachyceras (1.665). Maytenus obtusifolia é abundante 
nas restingas do Rio de Janeiro, inclusive, é a espécie que apresenta maior valor de importância na porção florestal do Complexo Lagunar Grussai/lquipari (RJ) (Assumpção; Nascimento, 2000; Pereira, 2001).

\section{Espécies Ameaçadas}

Dentre as 78 espécies registradas no Horto Restinga, 15 estão ameaçadas de extinção de acordo com as fontes consultadas (Tabela 2). Considerando apenas o nível municipal, são nove espécies na categoria "Vulnerável" e três na categoria "Em Perigo". Há duas espécies ameaçadas a nível nacional. Quanto ao status internacional, uma espécie está "Em Perigo" enquanto que cinco são consideradas de baixo risco.

Tabela 2: Espécies ameaçadas de extinção registradas no Horto Restinga, segundo as listas da União Internacional para a Conservação da Natureza (IUCN), Instituto de Pesquisas Jardim Botânico do Rio de Janeiro (JBRJ), Ministério do Meio Ambiente (MMA) e Secretaria do Meio Ambiente DO Município do Rio de Janeiro (SMAC-SP). CR, Criticamente em Perigo; EN, Em Perigo; VU, Vulnerável; LR, Baixo Risco.

\begin{tabular}{|c|c|c|c|c|}
\hline \multirow{2}{*}{ Família/Espécie } & \multicolumn{4}{|c|}{ Status de ameaça } \\
\hline & SMAC & MMA & IUCN & JBRJ \\
\hline \multicolumn{5}{|l|}{ APOCYNACEAE } \\
\hline Aspidosperma parvifolium & VU & & & \\
\hline \multicolumn{5}{|l|}{ BROMELIACEAE } \\
\hline Vriesea neoglutinosa & EN & & & \\
\hline \multicolumn{5}{|l|}{ BURSERACEAE } \\
\hline Protium icicariba & & EN & & EN \\
\hline \multicolumn{5}{|l|}{ CLUSIACEAE } \\
\hline Clusia fluminensis & VU & & & \\
\hline Kielmeyera membranacea & VU & & & \\
\hline \multicolumn{5}{|l|}{ FABACEAE } \\
\hline Andira legalis & VU & & & \\
\hline Inga maritima & VU & VU & EN & VU \\
\hline Ormosia arborea & VU & & & \\
\hline Senna pendula & & & LR & \\
\hline Zollernia glabra & VU & & LR & \\
\hline \multicolumn{5}{|l|}{ MORACEAE } \\
\hline Ficus hirsuta & EN & & LR & \\
\hline \multicolumn{5}{|l|}{ MYRTACEAE } \\
\hline Myrrhinium atropurpureum & VU & & & \\
\hline
\end{tabular}




\begin{tabular}{ccc} 
RUTACEAE & & \\
Esenbeckia grandiflora & EN & \\
SAPOTACEAE & & LR \\
Manilkara subsericea & VU & LR \\
Pouteria grandiflora & & \\
\hline
\end{tabular}

Das espécies listadas, 08 estão ameaçadas de extinção apenas no município do Rio de Janeiro. Grande parte das espécies ameaçadas apresenta distribuição geográfica restrita e são afetadas pela degradação das restingas da cidade do Rio de Janeiro.

As espécies consideradas de "baixo risco" pela Lista Vermelha da IUCN, são consideradas estáveis até o presente momento, e são comumente encontradas em áreas protegidas. Contudo, são espécies que requerem atenção ao longo dos próximos anos para que não se enquadrem em categorias de maior risco de extinção.

A espécie Protium icicariba é classificada como "Em Perigo" pelas duas listas nacionais consideradas, apesar de não se encontrar na lista do município e ser considerada uma espécie de ocorrência limitada ao estado da Bahia pelo Livro vermelho da flora do Brasil (2013), a Protium icicariba também é encontrada no Espírito Santo e Rio de Janeiro. Segundo Ferreira et al (2010), a espécie apresentou frequência de $90 \%$ em relação as parcelas analisadas na Área de Proteção Ambiental de Setiba no Espírito Santo, em que a densidade populacional foi de 7,4 indivíduos $/ \mathrm{m}^{2}$. Além disso, a espécie foi registrada por Castro et al. (2007) na formação arbustiva aberta não inundável da Restinga da Marambaia - RJ; sendo classificada como a terceira espécie em valor de importância em relação as demais 29 espécies registradas no local, e no geral, é uma espécie encontrada nas restingas do município do Rio de Janeiro, inclusive, produzida como mudas para reflorestamento (Zamith; Scarano, 2004).

Protium icicariba é uma espécie arbustiva que ocorre em floresta ombrófila densa e restinga, sua principal característica é a capacidade em exsudar resinas aromáticas, voláteis usadas com diversas finalidades. A folhas são compostas, podem apresentar de 5 a 7 folíolos, a textura das folhas é coriácea e apresenta coloração verde escura na face superior e mais clara na 
inferior. O fruto é uma cápsula de pericarpo avermelhado quando maduro, que expõe as sementes sarcotesta branca (Ferreira et al., 2010). Segundo Montezuma e Araújo (2007), a espécie Protium icicariba junta com a Humiria balsamifera são as que melhor representam a formação Arbustiva Inundável de restinga do município de Carapebus-RJ, sendo que a primeira despontou como a espécie de maior densidade, enquanto que $H$. balsamifera é a de maior dominância para a localidade em questão.

Apenas uma espécie, Inga maritima, consta como ameaçada simultaneamente nas listas que consideram os níveis mundial, nacional e municipal. A espécie Inga maritima pertence à família Fabaceae, é endêmica do estado do Rio de Janeiro. Apesar de ser considerada uma espécie ameaçada de extinção, é encontrada com significativa frequência nas restingas do estado do Rio de Janeiro, de acordo com Neves Filho (2009), esta espécie é comum nas restingas do tipo arbustivo fechado pós-praia e no tipo arbóreo não inundado nas áreas de restinga do bairro Barra da Tijuca, RJ.

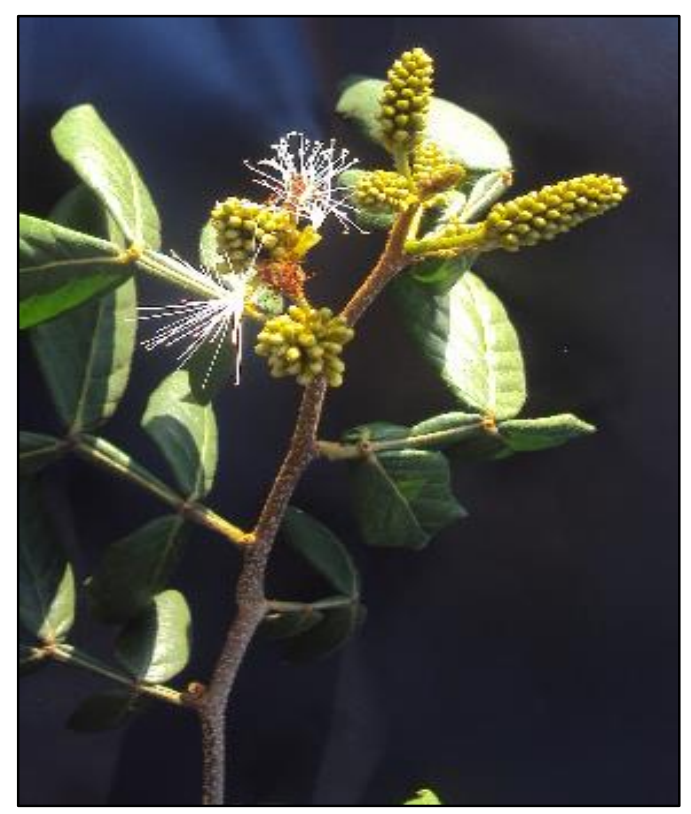

Figura 1: Floração de muda de Inga marítima do Horto Restinga.

O Inga maritima apresenta folhas compostas paripinadas, alternas, com estípulas caducas, pecíolo alado, piloso; raque alada. Pode atingir $2,5 \mathrm{~m}$ de altura. Para Silva (2012), as mudas desta espécie apresentam um crescimento satisfatório, e costumam ter baixa mortalidade na área alagada. Segundo 
Menezes e Araújo (2004), ao analisar a regeneração e riqueza da formação arbustiva de 3 sítios distintos, pós-fogo na Restinga da Marambaia-RJ, foi observado que a espécie em questão apresentou um dos três maiores valores de dominância relativa dos sítios estudados, além disso, apresentou elevado valor de importância. $\mathrm{E}$ as espécies com valores de importância altos costumam apresentar vantagens significativas relacionadas a rebrotação após o fogo.

A produção de mudas de espécies de restingas é essencial para o município, visto que muitas espécies de restingas são endêmicas do país e muitas estão ameaçadas de extinção, principalmente, a Inga marítima. Essas mudas podem ser destinadas a recuperação de áreas degradadas e a recomposição florestal visando o cumprimento de medidas compensatórias oriundas de autorizações de supressão vegetal no município do Rio de Janeiro.

\section{CONCLUSÃO}

É de extrema importância a produção de mudas de espécies de restingas no município do Rio de Janeiro. No Horto Restinga foram contabilizados, no mês de agosto de 2015, 47.390 mudas distribuídas em 78 espécies e 30 famílias botânicas. As famílias Fabaceae e Myrtaceae são as mais representativas no horto. Dentre as espécies observadas, 15 espécies são consideradas ameaçadas de extinção em pelo menos uma das bibliografias consultadas.

\section{REFERÊNCIAS}

AFONSO, A.S.; MEDEIROS, A.S.; NUNES, C.S.; RODRIGUES, G.A.; NUNES, R.S.; TAVARES, L.F.M.; CONDE, M.M.S. Florística da vegetação arbustiva aberta na Restinga da Marambaia, RJ. Revista Brasileira de Biociências, Porto Alegre, v. 5, n. 2, p. 450-452, 2007.

ALONSO, J.M.; LELES, P. S.S.; FILHO, T. B. S.; MESQUITA, C.A.B.; PEREIRA, M.L.; SALES JUNIOR, A.SS.; ALVES, F.L.; SILVA, C.O.Avaliação da diversidade de espécies nativas produzidas nos viveiros florestais do estado do Rio de Janeiro. FLORESTA, Curitiba, PR, v. 44, n. 3, p. 369 - 380, 2014. 
ALVES, L.F.; METZGER, J.P. A regeneração florestal em áreas de floresta secundária na reserva Florestal do Morro Grande, Cotia, SP. Biotaneotropica, v.6, n.2, p. 1-26, 2006

ASSUMPÇÃO, J.; NASCIMENTO, M. T. Estrutura e composição florística de quatro formações vegetais de restinga no complexo lagunar Grussaí/lquipari, São João da Barra, RJ, Brasil. Acta Botanica Brasilica, São Paulo, v. 14, n.3, p.301-315, 2000.

BARBOSA, L. M. ; BARBOSA, J. M. ; BARBOSA, K. C. ; POTOMATI, A. ; MARTINS, S. E. ; ASPERTI, L. M. ; MELO, A. C. G. ; CARRASCO, P. G. ; CASTANHEIRA, S. A. ; PILIACKAS, J. M. . Recuperação florestal com espécies nativas no Estado de São Paulo: pesquisas apontam mudanças necessárias. Florestar Estatístico, v. 6, p. 28-34, 2003.

CNCFLORA. Base de Dados do Centro Nacional da Conservação da Flora. Disponivel em: <http://cncflora.jbrj.gov.br/portal/>. Acesso em:7. Ago. 2015.

BRASIL. Ministério do Meio Ambiente, Conselho Nacional do Meio Ambiente (CONAMA), Resolução 303. Brasília. 2002. Conselho Nacional do Meio Ambiente. Disponível em: <http://www.mma.gov.br/port/conama/res/res02/res30302>. Acesso em: 6 ago. 2015.

CARRASCO, P. G.; CASTANHEIRA, S.A.; BARBOSA, L.M.; OLIVEIRA, M.A.; COUTINHO, L.F. Produção e avaliação do crescimento de mudas de espécies florestais de restinga, com e sem adubação. In: CONGRESSO INTERNACIONAL DE TECNOLOGIAS PARA O MEIO AMBIENTE, 3, 2012. Bento Gonçalves. Resumos... Bento Gonçalves, RS. Universidade de Caxias do Sul, 2012.

CASTRO, D. N.; SOUZA, M.; MENEZES, L.F.T. Estrutura da formação arbustiva aberta não inundável na Restinga da Marambaia, RJ. Revista Brasileira de Biociências, Porto Alegre, v. 5, n. 2, p. 75-77, 2007

FALKENBERG, D. B. Aspectos da Flora e da vegetação secundária da restinga de Santa Catarina, sul do Brasil. Insula, n.28: 1-30. 1999.

FERREIRA L.C., THOMAZI R.D., OLIVEIRA D.A.C., SILVA A.G. Estrutura populacional e padrão espacial de Protium icicariba (DC.) Marchand na Área de 
Proteção Ambiental de Setiba, Espírito Santo, sudeste do Brasil. Natureza on line, Santa Tereza, v.8, n.1, p.39-45, 2010.

GARCIA, F.C.P., FERNANDES, J.M. Inga in Lista de Espécies da Flora do Brasil, Jardim Botânico do Rio de Janeiro. Jardim Botânico do Rio de Janeiro. Disponivel em: <http://floradobrasil.jbrj.gov.br/2012/FB022803>.Acessoem: 5 Ago. 2015.

GENTRY, A.H. Endemism in tropical versus temperate plant communities. In M.E. SOULÉ (ed.) Conservation biology, the science and diversity. Sinauer Associates. Sunderland, p. 153-181.1986.

GOMES, J.M.; COUTO, L.; BORGES, R. de C.G. et al. Efeitos de deferentes substratos na produção de mudas de Eucalyptus grandis W. Hill ex Maiden, em "win-strip". Revista Arvore, Viçosa, v.15, n.1, p.35-42, 1991.

GOMES, F. H.; TORRADO, P. V.; MACÍAS F.; GHERARDI, B.; PEREZ, X.L.O. Solos Sob Vegetação De Restinga Na llha Do Cardoso (Sp). I - Caracterização e Classificação. R. Bras. Ci. Solo, vol.31, n.6, pp. 1563-1580, 2007.

KRUCKEBERGER, A.R. \& RABINOWITZ, D. Biological aspects of endemism in higher plants. Ann. Rev. Ecol. Syst, v.16, p.447-479. 1985.

Lista de Espécies da Flora do Brasil. Jardim Botânico do Rio de Janeiro. Disponível em: <http://floradobrasil.jbrj.gov.br/>. Acesso em: 10 Ago. 2015.

Lista de Espécies da Flora Ameaçadas de Extinção na Cidade do Rio de Janeiro. Secretaria do Municipal do Meio Ambiente-RJ. Decreto № 19.149. 2000.

Lista Nacional Oficial de Espécies da Flora Ameaçadas de Extinção. Ministério do Meio Ambiente. Portaria MMA no 443, 2014.

MARTINELLI, G.; MORAES, M.A.; ANDERSON, F.; HIEATT, C.Livro Vermelho da flora do Brasil. - 1. ed. - Rio de Janeiro : Instituto de Pesquisas Jardim Botânico do Rio de Janeiro, 2013.

MENEZES, L.F.T.; ARAUJO, D.S.D. Regeneração e riqueza da formação arbustiva de Palmae em uma cronosequência pós-fogo na Restinga da 
Marambaia, Rio de Janeiro, RJ, Brasil. Acta Bot. Bras., São Paulo, v.18, n.4, 2004.

OLIVEIRA M. A. M. Potencial de regeneração de um fragmento de Floresta Baixa de Restinga, em Ilha Comprida, SP, degradado para cultivo agrícola Dissertação apresentada ao Instituto de Botânica da Secretaria de Estado do Meio Ambiente. São Paulo, 2014.

PEREIRA, M. C.A.; ARAUJO, D.S.D.; PEREIRA, O.J. Estrutura de uma comunidade arbustiva da restinga de Barra de Maricá - RJ. Revista Brasil Bot., São Paulo, V.24, n.3, p.273-281, 2001

RIBEIRO, R.D.; LIMA, H.C. Riqueza e distribuição geográfica de espécies arbóreas da familia Leguminosae e implicações para conservação no Centro de Diversidade Vegetal de Cabo Frio, Rio de Janeiro, Brasil. Rodriguesia, v.60, n.1, p.111-127, 2009.

RIZZINI, C. T. Tratado de fitogeografia do Brasil. 2ed. Âmbito Cultural Edições Ltda, Rio de Janeiro, p. 327. 1997.

RODRIGUES, R. R. Recuperação de áreas degradadas em restinga. In: BARBOSA, L. M. (Coord.). Recuperação de áreas degradadas da serra do mar e formações florestais litorâneas. São Paulo: Secretaria do Meio Ambiente, p. $98-105,2000$.

RODRIGUES, M.A.; PAOLI, A.A.S.; BARBOSA, J.M.; SANTOS JUNIOR, N.A.Avaliação da chuva de sementes em áreas de restinga em diferentes estágios de regeneração. Revista Árvore, Viçosa-MG, v.34, n.5, p.815-824, 2010

SILVA, G.D.F. Avaliação do estabelecimento de mudas nativas em áreas de restauração ecológicas na RPPN Caruara - Restinga do Complexo Lagunar Grussaí-lquipari, RJ. 2012. Monografia (Graduação em Ciências Biológicas) Universidade Estadual do Norte Fluminense Darcy Ribeiro, Campos dos Goytacazes.

SILVEIRA-FILHO, T. B. A política florestal estadual do Rio de Janeiro: ação e inação do estado entre 1975-2011. 150 f. Dissertação (Mestrado em Ciências Ambientais e Florestais) - Universidade Federal Rural do Rio de Janeiro, Seropédica, 2012. 
STILE, E.W. Fruits, seeds and dispersal agents. In: Abraham, W.G. Plant animal interactions. Mc Graw Hill, New York. 1989. 351 p.

The IUCN Red List of Threatened Species. Version 2015.2. Disponível em: <www.iucnredlist.org >. Acesso em: 10 ago 2015.

ZAMITH, L. R. SCARANO, F.R. Produção de mudas de espécies das Restingas do município do Rio de Janeiro, RJ, Brasil. Acta bot. Bras. São Paulo, v.18, n.1, p.161-176. 2004.

Recebido:13/09/2015

Aprovado:28/11/2015 